Supporting Information for:

\title{
Combination of thin-layer chromatography and mass spectrometry using cluster-induced desorption/ionization
}

Julian Heep ${ }^{1}$, Paul H. K. Tuchecker ${ }^{1}$, Christoph R. Gebhardt ${ }^{2}$, and Michael Dürr ${ }^{1, *}$

${ }^{1}$ Institut für Angewandte Physik, Justus-Liebig-Universität

Giessen, D-35392 Giessen, Germany

${ }^{2}$ Bruker Daltonik GmbH, D-28359 Bremen, Germany

${ }^{*}$ Corresponding author: michael.duerr@ap.physik.uni-giessen.de (M.D.) 
Assignment of peaks in mass spectra from egg yolk lipids

\begin{tabular}{|c|c|}
\hline$m / z$ value & assignment \\
\hline 520.5 & LPE 18:0 $+\mathrm{K}^{+}$ \\
\hline 703.6 & $\mathrm{SM} 16: 0+\mathrm{H}^{+}$ \\
\hline 725.5 & $\mathrm{SM} 16: 0+\mathrm{Na}^{+}$ \\
\hline 756.5 & PE 16:0/18:1 $+\mathrm{K}^{+}$ \\
\hline 760.5 & PC 16:0 18:1 $+\mathrm{H}^{+}$ \\
\hline 782.5 & PC 16:0 18:1 $+\mathrm{Na}^{+}$ \\
\hline 788.6 & PC 18:0 18:1 + $\mathrm{H}^{+}$ \\
\hline 810.6 & PC 18:0 18:1 $+\mathrm{Na}^{+}$ \\
\hline 835.6 & PI 16:0/18:2 $+\mathrm{H}^{+}$ \\
\hline 873.6 & PI 17:0/18:0 $+\mathrm{Na}^{+}$ \\
\hline 879.6 & PI 16:0/18:1-H $\mathrm{H}^{+}+2 \mathrm{Na}^{+}$ \\
\hline 881.7 & PI 16:0/18:0-H $\mathrm{H}^{+}+2 \mathrm{Na}^{+}$ \\
\hline 893.7 & PI 17:0/18:1- $\mathrm{H}^{+}+2 \mathrm{Na}^{+}$ \\
\hline 895.7 & PI 17:0/18:0 - $\mathrm{H}^{+}+2 \mathrm{Na}^{+}$ \\
\hline 901.6 & PI 18:0/18:2 $+\mathrm{K}^{+}$ \\
\hline 907.6 & PI 18:0/18:2-H $\mathrm{H}^{+}+2 \mathrm{Na}^{+}$ \\
\hline 909.6 & PI 18:0/20:4 $+\mathrm{Na}^{+}$ \\
\hline 911.6 & PI 18:0/20:3 $+\mathrm{Na}^{+}$ \\
\hline
\end{tabular}

Table S1: Assignment of the peaks identified in the spectra shown in Fig. 5 according to Refs. [6,26-28]. 